Edited by Kiriakos Xenitidis and
Colin Campbell
Contents
- Is depression one thing or many?
- CBT for psychosis: not a 'quasi-neuroleptic'
- Stimulant treatment for ADHD
- Liaison services for older adults

\section{Is depression one thing or many?}

Until 1980, manic depressive illness (MDI) was defined as follows: the presence of recurrent severe depressive or manic episodes alternating with periods of normal mood or markedly decreased mood symptoms. ${ }^{1}$ In that year, the DSM-III rejected this definition of MDI and replaced it with two different conditions: bipolar disorder and major depressive disorder (MDD). Bipolar disorder meant the presence of manic and depressive episodes, not manic or depressive episodes - a huge difference between bipolar disorder and MDI. Recurrent episodic severe depression, previously called MDI, was redefined as MDD, which also included other varieties of depressive symptoms (such as neurotic depression). In the last few decades, the broad heterogeneous definition of MDD has been accepted by many clinicians as if it was a single entity, completely different from bipolar disorder, without awareness that both conditions were seen as one illness in the past: MDI.

We present the case of a patient with severe recurrent depression who achieved clinical remission after treatment with lithium monotherapy despite non-efficacy of standard antidepressants.

The patient was a 36 year-old White, married woman with a positive family history for bipolar disorder. She had no history of manic episodes.

At 32 years of age, 2 weeks after delivering her first child, she presented feelings of inadequacy and guilt, anhedonia and decreased appetite. She had difficulty falling asleep because of ruminative pessimistic thoughts about the future. These symptoms met DSM criteria for a major depressive episode and improved in the next few weeks without medications.

Six months later, anhedonia increased prominently and she presented depressed mood, feelings of worthlessness and occasional suicidal thoughts. She was admitted to the in-patient service and was treated with sertraline $250 \mathrm{mg}$ /day.

In the following 2 years she had three depressive episodes per year lasting about 2 months each without full interepisode recovery. Her treatment was modified to clomipramine $225 \mathrm{mg} /$ day. Depressive episodes recurred more frequently and lasted about 1 month each.

At age 35, she was admitted to the in-patient service after impulsive ingestion of high doses of benzodiazepine and antidepressants as a suicide attempt. Clomipramine was gradually discontinued and treatment was modified to lithium $600 \mathrm{mg} /$ day (serum level $0.5-0.7 \mathrm{mEq} / \mathrm{l}$ ). Lithium was maintained in monotherapy; depressive symptoms were well controlled and there were no mood recurrences for the following 12 months.

In this case, four features support the hypothesis of underlying MDI: presence of recurring depressive episodes, positive family history for bipolar disorder, postpartum onset of first depressive episode, and improvement of affective symptoms with a mood stabiliser. Randomised studies indicate that standard therapeutic levels of lithium are effective in recurrent depressive episodes, whether bipolar or unipolar, in both acute and maintenance treatment phases. ${ }^{2,3}$

DSM-III made a radical change in dividing the broad MDI concept into the narrow bipolar and broad MDD concepts. Given decades of research suggesting that MDD is a heterogeneous concept, and the perspective that course (recurrence of mood episodes) may be highly important in diagnosis, rather than symptoms (depression v. mania), ${ }^{4}$ case examples such as this one raise the question whether therapeutic response also supports a return to the MDI concept that emphasised diagnosis based on recurrence of episodes, irrespective of depressive or manic polarity.

This nosological approach would have important clinical implications if MDI is the disease that produces both depressive and manic symptoms. William Osler's view that the medical profession should primarily treat diseases, not symptoms, ${ }^{5}$ would imply that mood stabilisers such as lithium should be the main long-term treatment of choice in patients with recurrent depression. Addition of low doses of antidepressants could be considered for short-term symptom improvement, rather than long-term prevention of mood episodes, while monitoring emerging manic or mixed episodes.

\section{Declaration of interest}

S.N.G. has provided research consulting to Sunovion and Pfizer, and has obtained a research grant from Takeda Pharmaceuticals.

1 Ghaemi SN, Vohringer PA, Whitham EA. Antidepressants from a public health perspective: re-examining effectiveness, suicide, and carcinogenicity. Acta Psychiatr Scand 2013; 127: 89-93.

2 Cipriani A, Smith K, Burgess S, Carney S, Goodwin G, Geddes J. Lithium versus antidepressants in the long-term treatment of unipolar affective disorder. Cochrane Database Syst Rev 2006; 4: CD003492.

3 Bschor $\mathrm{T}$, Uhr $\mathrm{M}$, Baethge $\mathrm{C}$, Lewitzka $\mathrm{U}$, Ising $\mathrm{M}$, Erbe $\mathrm{S}$, et al. Acute antidepressive efficacy of lithium monotherapy, not citalopram, depends on recurrent course of depression. J Clin Psychopharmacol 2013; 33: 38-44.

4 Smith DJ, Craddock N. Unipolar and bipolar depression: different or the same? Br J Psychiatry 2011; 199: 272-4.

5 Ghaemi SN. Hippocratic psychopharmacology for bipolar disorder - an expert's opinion. Psychiatry 2006; 3: 30-9.

A. Amerio, Section of Psychiatry, Department of Neuroscience, University of Parma, c/o Ospedale Maggiore, Pad. 21 - Braga, Viale A. Gramsci 14, 43126 Parma, Italy. Email: andrea.amerio@studenti.unipr.it; A. Odone, school of Medicine-Public Health Unit, University of Parma, Parma, Italy, and Department of Global Health and Social Medicine, Harvard Medical School, Boston, Massachusetts, USA; C. Marchesi,

Department of Neuroscience, Section of Psychiatry, University of Parma, Parma, Italy; S. N. Ghaemi, Mood Disorders Program, Tufts Medical Center, and Tufts University Medical School, Department of Psychiatry and Pharmacology, Boston, Massachusetts, USA

doi: $10.1192 /$ bjp.204.6.488

\section{CBT for psychosis: not a 'quasi-neuroleptic'}

As members of the recent National Institute for Health and Care Excellence (NICE) clinical guideline update for schizophrenia ${ }^{1}$ (M.B. and D.S.), we read with interest the excellent meta-analysis of CBT for symptoms of schizophrenia by Jauhar et al. ${ }^{2}$ The results are broadly in line with the NICE review and particularly that of Wykes et al, ${ }^{3}$ which showed that studies with high methodological rigour, including masking, have a small effect size for positive and total symptoms. Clearly cognitive-behavioural therapy (CBT) is no panacea; but neither is it ineffective. Meta-analyses bring together all trials, with patients drawn from heterogeneous populations, including different phases of illness. The tests for 